\title{
The future vision of simulation in health care
}

\section{M Gaba}

Simulation is a technique - not a technology - to replace or amplify real experiences with guided experiences that evoke or replicate substantial aspects of the real world in a fully interactive manner. The diverse applications of simulation in health care can be categorised by 11 dimensions: aims and purposes of the simulation activity; unit of participation; experience level of participants; health care domain; professional discipline of participants; type of knowledge, skill, attitudes, or behaviours addressed; the simulated patient's age; technology applicable or required; site of simulation; extent of direct participation; and method of feedback used. Using simulation to improve safety will require full integration of its applications into the routine structures and practices of health care. The costs and benefits of simulation are difficult to determine, especially for the most challenging applications, where long term use may be required. Various driving forces and implementation mechanisms can be expected to propel simulation forward, including professional societies, liability insurers, health care payers, and ultimately the public. The future of simulation in health care depends on the commitment and ingenuity of the health care simulation community to see that improved patient safety using this tool becomes a reality.

Correspondence to: Professor David M Gaba, Patient Simulation Center of Innovation at VA Palo Alto Health Care System, Center for Immersive and Simulation-based Learning, Stanford University, Anesthesia Service, 112A, VA Palo Alto HCS, 3801 Miranda Avenue, Palo Alto, CA 94304, USA; gaba@stanford.edu
$\mathrm{T}$ he past two decades-and especially the last five years-have seen rapidly growing interest in using simulation for purposes of improving patient safety and patient care through a variety of applications. Simulation is a technique, not a technology, to replace or amplify real experiences with guided experiences, often immersive in nature, that evoke or replicate substantial aspects of the real world in a fully interactive fashion. "Immersive" conveys the sense that participants have of being immersed in a task or setting as they would if it were the real world. The ideal example of full immersion (admittedly fictional) would be the Star Trek "holodeck", in which one literally cannot tell the difference between the simulated experience and real life. While such seamless immersion is not currently achievable, experience shows that participants in immersive simulations easily suspend disbelief and speak and act much as they do in their real jobs.

While this definition encompasses a wide variety of experiential activities, the term "simulator" as used in health care usually refers to a device that presents a simulated patient (or part of a patient) and interacts appropriately with the actions taken by the simulation participant. The interest in simulation for health care has derived in large measure from the long experience and heavy use of simulation for training and other purposes in non-medical industries. In particular, these include commercial aviation, nuclear power production, and the military-industries that share with health care intrinsic hazard and complexity, but are considered high reliability organisations that have a very low failure rate considering their inherent risks. ${ }^{1-3}$ Health care simulators are directly analogous to the flight simulators that have become well known to the public.

This objectives of this paper are: to provide a comprehensive framework for understanding the diversity of applications of simulation in health care, as categorised by 11 different dimensions; to provide a vision of how fully integrating simulation into the structures and processes of health care can be used to revolutionise patient care and patient safety; and to provide an overview of the driving forces and implementation mechanisms by which different entities may, or may not, promulgate simulation over the next 20 years. Although written to some degree from the perspective of the USA, the issues discussed are similar throughout the world.

\section{HOW SIMULATION CAN IMPROVE PATIENT SAFETY}

Those working on the development and use of simulation in health care largely share a common vision of a future revolution in health care organisation, with simulation as a key enabling technique. We seek a model in which the structures and systems of health care are optimised for safety, quality, and-where it does not conflict with these goals-for efficiency. Current systems of health care throughout the world do not accomplish this (see, for example, the US Institute of Medicine reports on medical error $^{4}$ and on "crossing the quality chasm" ${ }^{\prime 5}$ ). The revolution that we envision concerns how personnel are educated, trained, and sustained for providing safe clinical care. Currently, the health care system places a premium on basic science education and leaves most clinical training to a relatively unsystematic apprenticeship process. The emphasis is on individual knowledge and skill rather than on honing the performance of clinical teams. Once a clinician has completed training, the required level of continuing education and training is often minimal and unstructured.

Thus a fundamental part of the vision for the future is that clinical personnel, teams, and 
systems should undergo continual systematic training, rehearsal, performance assessment, and refinement in their practice. This vision is inspired in part by the systems in place in various high reliability organisations, particularly commercial aviation, but it is not slavishly copied from their experiences. Needless to say, using simulation as part of the process of revolutionising health care is more complex than merely attempting to stick simulation training on top of the current system. Moreover, beyond training, simulation may provide indirect ways to improve safety, including facilitating recruitment and retention of skilled personnel, acting as a lever for culture change, and improving quality and risk management activities.

\section{SIMULATION APPLICATIONS ARE DIVERSE AND CAN BE CATEGORISED BY 11 DIMENSIONS}

Current and future applications can be categorised by 11 dimensions, each of which represents a different attribute of simulation (fig 1). For many dimensions there is a clear gradient and direction, although for others there are only categorical differences. Over the last 20 years, the demonstrated applications of simulation to health care have been quite diverse, and in fact some have been in use for decades, but the space of possible applications has not been fully examined. In the future, additional portions of the 11dimensional "application space" will be more extensively explored, weaving a rich and complex tapestry of simulation in health care. The total number of unique combinations across all the dimensions is very large (on the order of $5^{11}$ over 48 million-or more!), so clearly some combinations are either redundant or are not relevant.

\section{Dimension 1: The purpose and aims of the simulation activity}

The most obvious application of simulation is to improve the education and training of clinicians, but other purposes are also meaningful. Education emphasises conceptual knowledge, basic skills, and an introduction to the actual work. Training emphasises the actual tasks and work to be performed. Simulations can be used to assess performance and competency of individual clinicians and teams. Already, clinical skills examinations with standardised patient actors $^{67}$ are used in high stakes examinations. When applied to invasive and dangerous treatments in health care, only technological simulators can stand in for the patient. ${ }^{8-11}$

Simulation rehearsals are now being explored as adjuncts to actual clinical practice, for example where surgeons or an entire operative team can rehearse an unusually complex operation in advance using a simulation of the specific patient. ${ }^{12-14}$ Simulators can be powerful tools for research and evaluation, concerning organisational practices (patient care protocols) and for the investigation of human factors (for example, of performance shaping factors such as fatigue ${ }^{15}$ or of the user interface and operation of medical equipment in high hazard clinical settings ${ }^{16}{ }^{17}$ ). In fact, simulation based empirical tests of the usability of clinical equipment have already been used in designing equipment that is currently for sale; ultimately such practices may be required by regulatory agencies before approval of new devices.

Simulation can be a "bottom up" tool for changing the culture of health care to be more safety oriented, by training clinicians in practices that enact the desired "culture of safety". ${ }^{3}$ Simulation is also a rallying point about culture change and patient safety that can bring together experienced clinicians (who find the simulations clinically engaging) and health care administrators along with experts on human factors, organisational behaviour, or institutional change.

\section{Dimension 2: The unit of participation in the} simulation

Many simulation applications are targeted at individuals. These may be especially useful for teaching knowledge and basic skills. In other high hazard industries, such as aviation, individual skill is a fundamental building block, but a considerable emphasis is applied at higher organisational levels, in various forms of "crew resource management" (CRM). ${ }^{18}$ This is based on empirical findings that individual performance is not sufficient to achieve optimum safety. ${ }^{19} 20$ Team training may thus be addressed first to crews (also known as "single discipline teams"), consisting of multiple individuals from a single discipline, and then to teams (or "multidisciplinary teams" ${ }^{\prime 21}$ ). There are in fact advantages and disadvantages to addressing teamwork in the single discipline approach that "train crews to work in teams" versus the "combined team training" of multiple disciplines together. ${ }^{21}$ For maximum benefit these approaches may be used in a complementary fashion.

Teams exist in actual "work units" in an organisation (for example, a specific intensive care unit), each of which is its own target for training. Going further, there is also growing interest and experience in applying simulation to non-clinical personnel and work units in health care organisations (for example, to managers or executives) ) $^{22}$ and to the organisation as a whole (such as in disaster drills or in responding to a simulated catastrophic patient care accident).

\section{Dimension 3: The experience level of simulation participants}

Simulation can be applied from "cradle to grave" of clinical personnel. It can be used with early learners such as school children, or members of the lay public, to facilitate bioscience instruction, to interest students in biomedical careers, or to explain health care issues and practices. Clearly, the major role of simulation has been, and will continue to be, to educate, train, and provide rehearsal for those actually preparing for or working in the delivery of health care. Simulation is relevant from their earliest level of vocational or professional education (students), and during their apprenticeship training (for example, interns and residents). While these levels have been the main focus of simulation training to date, simulation is of growing importance for continuing training of experienced personnel, where-as in aviation-it can be applied regularly to practising clinicians (as individuals, teams, or organisations) regardless of their seniority. $\mathrm{z}^{23}{ }^{24}$ This approach provides an accumulation of experiences that are expected to have a long term synergism.

\section{Dimension 4: The health care domain in which the simulation is applied}

Simulation techniques can be applied across nearly all health care domains. Much of the attention on simulation has focused on technical and procedural skills applicable in surgery, ${ }^{13}{ }^{25-27}$ obstetrics, $^{28} 29$ invasive cardiology, ${ }^{30} 31$ and other related fields, while another bastion of simulation has been recreating whole patients for dynamic domains involving high hazard and invasive intervention, such as anaesthesia, ${ }^{32-36}$ critical care, ${ }^{37} 38$ and emergency medicine. . $^{39-42}$

While simulation might be applicable to image interpretation fields like radiology and pathology, in these domains personnel can often train using archived images of real patients, which is not an option in other domains. Where imaging is combined with invasive interventions, simulation can clearly be beneficial. ${ }^{43}$ In many domains, simulation techniques can be useful for addressing non-technical skills 


\begin{tabular}{|cccc|}
\hline Dimension 1: The purpose and aims of the simulation activity & \\
\hline Education & $\begin{array}{c}\text { Performance } \\
\text { assessment }\end{array}$ & $\begin{array}{c}\text { Clinical } \\
\text { rehearsal }\end{array}$ & $\begin{array}{c}\text { Research } \\
\text { (Human factors) }\end{array}$ \\
\hline
\end{tabular}

\begin{tabular}{|cccccc|}
\hline Dimension 2: The unit of participation in the simulation & & \\
\hline Individual & Crew & Team & Work unit & Organisation \\
\hline
\end{tabular}

Dimension 3: The experience level of simulation participants

\begin{tabular}{|c|c|c|c|c|}
\hline $\begin{array}{l}\text { School } \\
\text { Primary } \\
\text { Secondary }\end{array}$ & $\begin{array}{l}\text { College; } \\
\text { university }\end{array}$ & $\begin{array}{l}\text { Initial professional } \\
\text { education }\end{array}$ & $\begin{array}{c}\text { Residency or } \\
\text { on-the-job training }\end{array}$ & $\begin{array}{l}\text { Continuing education } \\
\text { and training }\end{array}$ \\
\hline
\end{tabular}

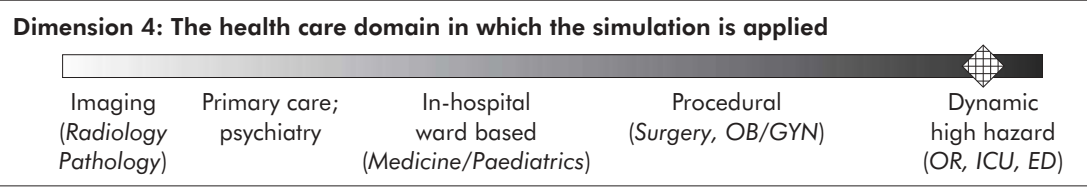

Dimension 5: The health care discipline of personnel participating in the simulation

\begin{tabular}{|c|c|c|c|c|c|}
\hline $\begin{array}{l}\text { Aids; } \\
\text { clerks }\end{array}$ & $\begin{array}{l}\text { Allied health; } \\
\text { technicians }\end{array}$ & $\begin{array}{c}\text { Nurses } \\
\text { (Including advanced } \\
\text { practice nurses) }\end{array}$ & Physicians & $\begin{array}{l}\text { Managers; } \\
\text { executives; } \\
\text { trustees }\end{array}$ & $\begin{array}{l}\text { Regulators; } \\
\text { legislators }\end{array}$ \\
\hline
\end{tabular}

Dimension 6: The type of knowledge, skill, attitudes, or behaviour addressed in simulation

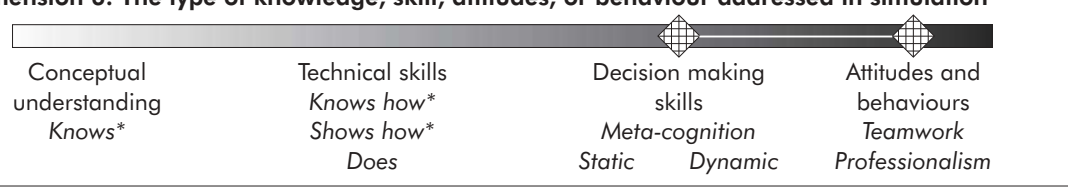

Dimension 7: The age of the patient being simulated

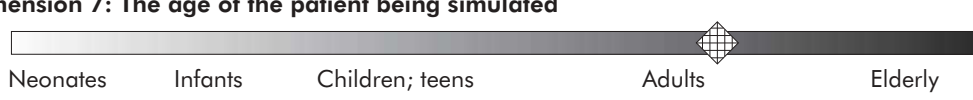

\begin{tabular}{|c|c|c|c|c|}
\hline \multicolumn{5}{|c|}{ Dimension 8: The technology applicable or required for simulations } \\
\hline $\begin{array}{l}\text { Verbal } \\
\text { Role playing }\end{array}$ & $\begin{array}{l}\text { Standardised } \\
\text { patients } \\
\text { (Actor) }\end{array}$ & $\begin{array}{c}\text { Part-task trainer } \\
\text { Physical; } \\
\text { virtual reality }\end{array}$ & $\begin{array}{l}\text { Computer patient } \\
\text { Computer screen; } \\
\text { screen based } \\
\text { "virtual world" }\end{array}$ & $\begin{array}{l}\text { Electronic patient } \\
\text { Replica of clinical site; } \\
\text { mannequin based; } \\
\text { full virtual reality }\end{array}$ \\
\hline
\end{tabular}

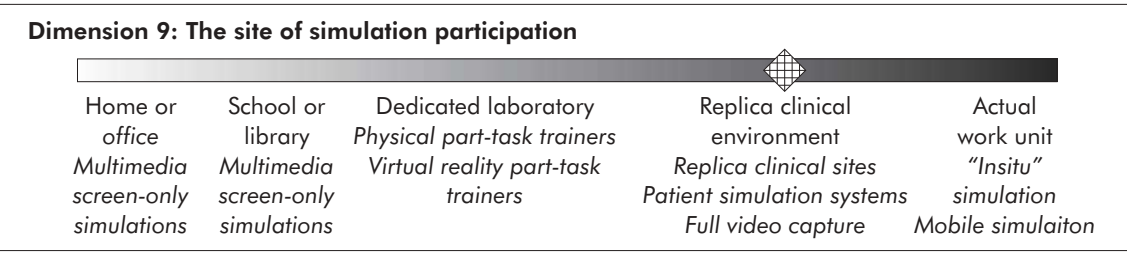

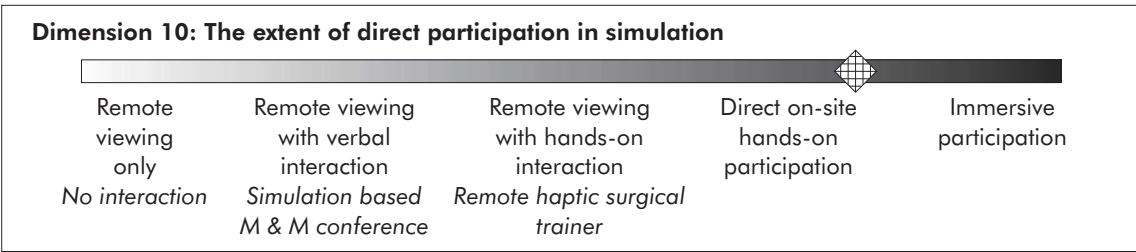

\begin{tabular}{|c|c|c|c|c|}
\hline \multicolumn{5}{|c|}{ Dimension 11: The feedback method accompanying simulation } \\
\hline None & $\begin{array}{l}\text { Automatic critique } \\
\text { by simulator } \\
\text { Real time } \\
\text { Delayed }\end{array}$ & $\begin{array}{l}\text { Instructor critique of } \\
\text { records of prior } \\
\text { simulation sessions }\end{array}$ & $\begin{array}{l}\text { Real time } \\
\text { critique } \\
\text { Pause/restart } \\
\text { Real time } \\
\text { mentoring }\end{array}$ & $\begin{array}{c}\text { Video based } \\
\text { post-hoc } \\
\text { debriefing } \\
\text { Individual/group }\end{array}$ \\
\hline
\end{tabular}

Figure 1 The 11 dimensions of simulation applications. Items marked with an asterisk are derived in part from miller. ${ }^{44}$ Any particular application of simulation can be categorised as a point or range in each dimension (shown by diamonds). The diamonds in this figure illustrate one specific application-multidisciplinary CRM oriented decision making and teamwork training for ICU personel. 
such as communicating with patients and coworkers, or in addressing issues such as ethics or end of life care.

\section{Dimension 5: The health care disciplines of personnel participating in the simulation}

Simulation is applicable to all disciplines of health care, not only to physicians. Thus simulation techniques are being used for nurses, and ultimately also for technicians, aids, and even clerical personnel, especially when training as a team or work unit. Nor is simulation limited to clinical personnel. It may also be directed at managers, executives, hospital trustees, regulators, and legislators. For these groups, simulation can convey the complexities of clinical work, and it can be used to exercise and probe the organisational practices of clinical institutions at multiple levels.

\section{Dimension 6: The type of knowledge, skill, attitudes, or behaviour addressed in simulation}

Simulations can be used to help learners acquire new knowledge, and to better understand conceptual relations and dynamics. Already today physiological simulations allow students to watch cardiovascular and respiratory functions unfold over time and respond to interventions-in essence making textbooks, diagrams, and graphs "come alive." The next step on the spectrum is acquisition of skills to accompany knowledge. ${ }^{44}$ Some skills follow immediately from conceptual knowledge (such as cardiac auscultation), whereas others involve intricate and complex psychomotor activities (like basic surgical skills). Isolated skills must be assembled into a new layer of clinical practices. For example, an understanding of the concepts of general surgery cannot be combined only with basic techniques of dissecting and suturing or manipulation of instruments to create a capable laparoscopic surgeon. Basic skills must be integrated into actual clinical techniques, a process for which simulation may have considerable power, especially as it can readily provide experience with even uncommon anatomical or clinical presentations.

In the current health care system, for most invasive procedures novices at a task will typically first perform the task on a real patient, albeit under some degree of supervision. They climb the learning curve, working on patients with varying levels of guidance. Simulation offers the possibility of having novices practice extensively before they begin to work on real patients as supervised "apprentices."

In this way and others, simulation is applicable to clinicians throughout their careers to support lifelong learning. It can be used to refresh skills for procedures that are not performed often. Furthermore, knowledge, skills, and practices honed as individuals must be linked into effective teamwork in diverse clinical teams, which in turn must operate safely in work units and larger organisations. ${ }^{345-47}$ Perpetual rehearsal of responses to challenging events will be needed, as the team or organisation must be practiced in handling them as a coherent unit.

\section{Dimension 7: The age of the patient being simulated}

To date the bulk of simulators and simulation applications have been addressed to adult patients and clinical activities relevant to adult medicine. In part this reflects the challenge of building fully interactive mannequins and trainers for small patients. Yet in truth simulation is applicable to nearly every type and age of patient, again literally from "cradle to grave." Simulation may be particularly useful for paediatric patients and clinical activities, because neonates and babies have smaller physiological reserves than do most adults. ${ }^{48} 49$ Fully interactive paediatric patient simulators will soon become available, and virtual reality techniques will ultimately make it easier to provide a variety of simulated paediatric clinical activities.

\section{Dimension 8: The technology applicable or required} for simulations

To accomplish these goals a variety of technologies (including no technology) will be relevant for simulation. Verbal simulations ("what if" discussions) and standardised patient actors $^{50-52}$ require no technology but can effectively evoke or recreate challenging clinical situations. Similarly very low technology_even pieces of fruit or simple dolls_can stand in for skin and muscle for the initial training of some manual tasks. Certain aspects of even complex tasks and experiences can be recreated even with low tech means. For example, some education and training on teamwork can be accomplished with role playing, analysis of videos, or drills with simple mannequins. ${ }^{53}$

Ultimately though, learning and practising complex manual skills (for example, surgery, cardiac catheterisation), or practising the dynamic management of life threatening clinical situations that include risky or noxious interventions (such as intubation or defibrillation), can only be fully accomplished using either animals-which for reasons of both cost and issues of animal rights is becoming very difficult_or a technological means to recreate the patient and the clinical environment. Simulation technologies vary from relatively simple multimedia to different sorts of parttask trainers to simulators. A part-task trainer is a device that replicates limited aspects of a task, but does not present an integrated experience. A "patient simulator" is a system that presents a fully interactive patient and an appropriate clinical work environment in one of the following ways:

- In actual physical reality, using a patient mannequin ("a mannequin based simulator")

- On a computer screen only (a "screen based simulator")

- Using virtual reality (VR; a "virtual reality simulator") by which parts or all of the patient and environment are presented to the user through two or three dimensional visual and audio representations, with or without touch (haptics) to create a more "immersive" experience. A screen based simulator can be viewed as a very limited VR simulator. In addition, VR devices that replicate particular procedures (for example, laparoscopic surgery) in a fully interactive fashion, and that use replicas of actual tools, are also referred to as simulators, even though they do not present the full patient.

\section{Dimension 9: The site of simulation participation}

Some types of simulation-those that use videos, computer programs, or the Web-can be conducted in the privacy of the learner's home or office using their own personal computer. More advanced screen based simulators might need more powerful computer facilities available in the medical library. Part-task trainers and virtual reality simulators are best fielded in a dedicated skills laboratory. Mannequin based simulation can also be used in a skills laboratory, although the more complex recreations of actual clinical tasks require either a dedicated patient simulation centre with fully equipped replicas of clinical spaces, or the ability to bring the simulator into an actual work setting (in situ simulation). There are advantages and disadvantages to doing clinical simulations in situ versus in a dedicated centre. For example, using the actual site allows training of the entire unit with all its personnel, procedures, and equipment. On the other hand, there will at best be limited availability of actual clinical sites and the simulation activity may distract from real patient care work. The dedicated simulation centre is a more controlled and available environment, allowing more comprehensive recording of sessions, and imposing no distraction on real activities. For large scale simulations (such 
as disaster drills) the entire organisation becomes the site of training.

Video conferencing and advanced networking may allow even advanced types of simulation to be conducted remotely (see dimension 10 below). For example, the collaborative use of virtual reality surgical simulators in real time has already been demonstrated, even with locations that are separated by thousands of miles.

\section{Dimension 10: The extent of direct participation in simulation}

Most simulations-even screen based simulators or part-task trainers-were initially envisioned as highly interactive activities with significant direct "on site" hands on participation. However, not all learning requires direct participation. For example, some learning can take place merely by viewing a simulation involving others, as one can readily imagine being in the shoes of the participants. A further step is to involve the remote viewers either in the simulation itself or in debriefings about what transpired. Several centres have been using videoconferencing to conduct simulation based exercises, including morbidity and mortality conferences. ${ }^{54}$ Because the simulator can be paused, restarted, or otherwise controlled, the remote audience can readily obtain more information from the on site participants, debate the proper course of action, and discuss with those in the simulator how best to proceed.

\section{Dimension 11: The feedback method accompanying simulation}

Much as in real life, one can learn a great deal just from the experience itself, without any additional feedback. For most complex simulations, specific feedback is provided to maximise learning. On screen based simulators or virtual reality systems, the simulator itself can provide feedback about the participant's actions or decisions, ${ }^{55}$ particularly for manual tasks where clear metrics of performance are readily delineated. ${ }^{56}{ }^{57}$ More commonly, human instructors provide feedback for simulations. This can be as simple as having the instructor review records of previous sessions that the learner has completed alone. For many target populations and applications an instructor provides real time guidance and feedback to participants while the simulation is going on. Here too, the ability to start, pause, and restart the simulation can be valuable. For the most complex uses of simulation, especially when training relatively experienced personnel, the typical form of feedback is a detailed post-simulation debriefing session, often using audio-video recordings of the scenario. Waiting until after the scenario is finished allows experienced personnel to apply their collective skills without interruption but then allows them to see and discuss the advantages and disadvantages of their behaviours, decisions, and actions.

\section{INTEGRATING SIMULATION FULLY INTO THE HEALTH CARE SYSTEM}

Looking across all 11 dimensions, simulation is clearly applicable in many ways throughout the health care system. The major revolution enabled by simulation can only be achieved if the relevant applications are fully integrated into the routine fabric of health care delivery. With such embedding simulation will not be an "add on", as it is today; it will be a routine part of the everyday work environment. Students will expect to engage in simulations as part of achieving many of their learning goals. Clinical trainees (for example residents) will often undergo intensive simulation training on days when they would otherwise be performing clinical work. The focused attention and case salience possible using simulation should more than make up for the relative loss of actual case experience. This is now becoming of greater importance as the work hours of trainees are reduced (significantly in Europe and to a lesser extent in the USA). Experienced clinicians will expect periodically to spend a day in the simulator instead of their usual clinical pursuits. Continuing education will be transformed into lifelong learning embedded within systems of care, rather than being solely at the discretion, time, or cost of individual practitioners.

In health care, as opposed to the experience of other high reliability industries, simulation will probably never replace the primacy for training of the apprenticeship system of supervised work on real patients. Unlike airplanes or nuclear power plants, we do not design and build human beingsnor do we receive the official instruction manual! Patient care is intrinsically more complex, and requires more human empathy and connection, than do other high hazard activities that have used simulation. Simulation will be employed for those activities for which it is best suited, particularly for activities that are hazardous, involve uncommon or rare situations, or for which experiential learning is of greatest value. Finding the right mix of traditional learning, simulation based learning, and actual patient care experience is an important challenge.

Another revolutionary feature enabled by simulation is that training can in many cases be performed not just for a fixed duration or number of cases, but rather to specific criterion levels of competency for key aspects of knowledge, skill, and behaviour. ${ }^{56}$ Establishing these competency criteria across all aspects of health care will be a challenge, but simulation is an essential component both for the research to establish the assessment and criterion setting methodologies, and also to provide equivalent cases to different personnel and teams for testing competency. Most criterion level competency assessment will be carried out in a formative fashion both for trainees and experienced personnel. Still, a key aspect of high reliability organisations is that they ensure that individuals, crews, teams, and work units function at demonstrated levels of competent performance. Thus high stakes testing using simulation will ultimately be a feature of the simulation vision.

\section{COST}

The cost of implementing the various applications of simulation across all 11 dimensions varies widely. Cost depends greatly on the mix of target population, purpose of simulation, and technology used. It also depends on how educational and clinical organisations succeed in reorganising their structures of work to incorporate simulation based learning. Some forms of simulation are inexpensive and distributed (for example, screen based or web based simulations and part-task trainers). Low cost is particularly important for early learners of tasks and skills, where routine availability and the possibility of repeated practice are most valuable. Where simulation training replaces existing training (for example, as a substitute for animal laboratories) its relative cost will also be relatively low. At the highest endproviding new training curricula to experienced clinical teams or work units, using high fidelity scenarios-the costs are likely to be substantial. Yet it is exactly for these applications that the greatest potential is seen for improving patient safety, when comparing health care to other high reliability organisations ${ }^{3}{ }^{4}$

For trainees and experienced clinicians, time must be set aside from clinical work to allow dedicated training; this will probably impose significant costs because the current system provides little time for dedicated training not attached to clinical service. Yet here too in high reliability organisations training is accepted as part of the work, not an accessory to it, 
and the industry hires sufficient personnel to allow this happen.

\section{BENEFITS}

The benefits derived from the various applications of simulation will be much harder to measure than the costs. Safety gains are intrinsically difficult to assess, whereas the magnitude of the investments made are starkly apparent. ${ }^{3}$ Some benefits may be direct, stemming from immediately discernable improved performance of individuals and teams. This might result in efficiencies in care and reduced errors that more than offset the costs of simulation based training. Many benefits probably depend on long term cumulative synergies. This yields a "chicken and egg phenomenon." Long term benefits may be apparent only if simulation is applied consistently over a long period of time (which is probably the case in aviation). Yet most institutions in health care are cautious about committing themselves to long term implementation without a definitive evidence base to justify its application.

However, the current system of education, training, and maintenance of proficiency has itself never been tested rigorously to determine whether it achieves its stated goals; the high level reviews ${ }^{45}$ of the performance of the health care industry suggest that it does not. No high reliability organisation has had data equivalent to a randomised clinical trial proving the benefits of simulation training, despite decades of use and regular assessment of individual and team performance both in real work and during simulations. ${ }^{58} 59$ Pioneering centres in health care are starting to take the leap of long term application with less than absolute proof of benefit. Even greater leaps may be required in the future. Moreover, reaping the benefits from enhanced training also requires that the principles taught are fully reinforced in the real world.

Ultimately the various potential benefits and costs of simulation-the perceived "business case" —will play out in different ways for different parts of the 11-dimensional simulation space. The business case will also vary considerably between regions and countries, with different types of driving forces, payment systems, and economic strength.

\section{DRIVING FORCES AND IMPLEMENTATION MECHANISMS}

Table 1 details various societal entities and the driving forces that might entice them to push for the vision described above. It also summarises the different implementation mechanisms that might be available to each entity. Again, because different countries, regions, and states will have different structures of health care systems, the relevance or strength of these entities will vary greatly, as will their driving forces and implementation strategies. It is likely that there will be a mix of drivers and implementation mechanisms in any locale. This may be especially true in settings like the USA that lack a single payer for health care, and that have a decentralised system of governmental regulation.

\section{TWO POSSIBLE HISTORIES SEEN FROM THE YEAR 2025}

To see how all these possibilities might play out, imagine that the year is 2025, and consider how future historians might view the history of simulation in health care. Outlines of two possible histories are presented: in one, the vision described above is successfully achieved; in the other, only tiny fragments of this vision are realised despite the early promise and enthusiasm for simulation's applications in health care.
The optimistic view from 2025: successful integration of simulation throughout the fabric of health care

At the beginning of the 21 st century various driving forces coalesced to achieve the vision of simulation embedded in the fabric of care. The first movement was generated by medical and nursing educators and clinical faculty, translated first through individual departments, hospitals, and professional schools, and later by professional societies, programme accreditation review committees, and specialty boards. Although hesitant at first, from 2004 onward, there was steady progress. The Society for Medical Simulation (SMS) in the USA and the Society in Europe for Simulation Applied to Medicine (SESAM) provided technical and political leadership for the simulation community ${ }^{*}$. Individual schools, training programmes, and hospitals adopted systematic simulation training for certain domains and disciplines. Typical targets were students and trainees (for example residents), although continuing education for experienced personnel caught on, and hospitals began to train specific work units. A variety of professional organisations around the world took an early lead in promulgating simulation training, encouraging other professional societies to follow. In a few cases they acted because the evidence base provided robust proof of the benefits of specific applications of particular types of simulation; however, in many other cases, institutions and organisations acted with a limited evidence base given the high face validity of the simulation approaches being used, the difficulty of conducting definitive studies of their success, and continuing dissatisfaction with traditional approaches. In particular, they judged that long term cumulative effects of the application of simulation would yield important synergisms over long periods of time. For example, a prominent university started a revolutionary and widely acclaimed new professional school in which physicians, nurses, and allied health personnel were trained together in the classroom, in frequent and diverse simulations, and culminating in joint clinical training "on the wards." Ultimately, the experience with those who adopted simulation early convinced those waiting for further information, even when the formal evidence was limited.

An important driver was the public. Safety is fundamentally a political question. Compared with many threats to the world's peoples, safety in health care-or indeed in commercial aviation-is of minor significance compared with famine, disease, and war. And yet, in the industrialised world, just as the public has long demanded nearly perfect safety in aviation, they began to demand equivalent strides in health care safety. By 2010, owing to the continued occurrence of highly publicised cases of death or brain damage linked to significant errors by clinicians and organisations, the public became fully aware of how haphazard is the training and assessment of clinical personnel. They demanded changes to put health care on par with other industries. This trend was seized upon by malpractice attorneys (in countries where litigation is common), who developed novel theories of negligence based on the inability of health care institutions to ensure the safety of patients from error chains of accident evolution, or even to systematically assure the competence of personnel. Health care institutions turned to simulation as a means to respond to this challenge, and the liability insurers in turn provided incentives (discounts on premiums) to adopt simulation, and later disincentives (refusal of coverage) for those who did not adopt it. Ultimately, governmental regulators and non-governmental accrediting agencies (for example, the Joint Commission for the Accreditation of Healthcare Organizations-JCAHO-for hospitals in the

*See the web sites of SMS at www. socmedsim.org and SESAM at www. uni-mainz.de/FB/Medizin/Anaesthesie/SESAM/welcome 
Table 1 Driving forces and implementation mechanisms for full integration of simulation into health care

\begin{tabular}{|c|c|c|}
\hline Entity* & Driving forces & Implementation mechanisms \\
\hline Simulation societies and researchers & $\begin{array}{l}\text { - Promulgate simulation } \\
\text { - Improve care and patient safety }\end{array}$ & - Research, position papers, standards, guidelines \\
\hline Professional schools & $\begin{array}{l}\text { - Improve learning } \\
\text { - Competition with other schools }\end{array}$ & $\begin{array}{l}\text { - Curricula } \\
\text { - Instructor training }\end{array}$ \\
\hline Professional societies & $\begin{array}{l}\text { - Improve performance } \\
\text { - Avoid government regulation }\end{array}$ & $\begin{array}{l}\text { - Guidelines/standards } \\
\text { - Curricula, research }\end{array}$ \\
\hline $\begin{array}{l}\text { Professional or subspecialty licensing or } \\
\text { accrediting organisation }\end{array}$ & $\begin{array}{l}\text { - Improve performance } \\
\text { - Assure maintenance of competency } \\
\text { - Respond to public pressure }\end{array}$ & $\begin{array}{l}\text { - Required curricula } \\
\text { - Simulation based testing } \\
\text { - Guidelines/standards }\end{array}$ \\
\hline Health care organisations & $\begin{array}{l}\text { - Improve care and patient safety } \\
\text { - Improve efficiency, reduce cost } \\
\text { - Competition with other organisations }\end{array}$ & $\begin{array}{l}\text { - Required curricula } \\
\text { - Internal testing }\end{array}$ \\
\hline Funders of medical care & $\begin{array}{l}\text { - Reduce costs } \\
\text { - Reduce errors }\end{array}$ & $\begin{array}{l}\text { - Required curricula } \\
\text { - Guidelines/standards }\end{array}$ \\
\hline Liability insurers & $\begin{array}{l}\text { - Reduce claims payout } \\
\text { - Reduce claims }\end{array}$ & $\begin{array}{l}\text { - Discounts on premiums } \\
\text { - Required curricula to receive coverage }\end{array}$ \\
\hline Accrediting organisations & - Improve and ensure uniformity of care and patient safety & $\begin{array}{l}\text { - Voluntary programs } \\
\text { - Standards }\end{array}$ \\
\hline Government & $\begin{array}{l}\text { - Same as funders } \\
\text { - Respond to pressure from public }\end{array}$ & $\begin{array}{l}\text { - Laws and regulations } \\
\text { - Oversight of voluntary programmes }\end{array}$ \\
\hline Public & $\begin{array}{l}\text { - Improve care and patient safety } \\
\text { - Reduce "training" on patients } \\
\text { - Ensure uniform competence and proficiency of clinicians }\end{array}$ & $\begin{array}{l}\text { - Media attention } \\
\text { - Acceptance of voluntary programs } \\
\text { - Pressure for government action }\end{array}$ \\
\hline
\end{tabular}

USA) institutionalised these changes. Again, they first offered incentives for conducting simulation training but eventually required such training as a standard.

Medical device regulators, such as the Food and Drug Administration in the USA, first encouraged and then required the submission of data from simulations as part of the approval process for devices. This was particularly relevant for testing the usability of equipment and especially on the user interfaces of diagnostic and therapeutic equipment.

\section{The pessimistic view from 2025: simulation's dismal failure despite early promise}

At the beginning of the 21 st century there was considerable interest in reforming health care delivery and in particular the way that personnel and systems were prepared for and sustained in competency of knowledge, skills, and behaviours. Simulation was highly touted as offering advantages in addressing these issues, based in part on its apparent (though unproven) success in high reliability organisations. Nearly two decades of previous academic work had provided tantalising suggestions that simulation could be an effective tool. Proponents offered a vision for the future of an integrated use of simulation for individuals, crews, teams, work units, and organisations as part of a revolution in health care.

The initial excitement turned into a "tempest in a teapot." In 2025 simulation is still used intermittently in some fields of health care, especially for students and trainees, but it never caught on widely, and it has made nearly zero impact on the actual delivery of care. Several historical factors account for this.

The public proved more interested in access to care and cost of care than in quality or safety of care. Following the corollary to "Wildavsky's Law of Medical Money"60 they wished to "save money on everyone else's health care," while still expecting top results for themselves or their family. Thus they failed to demand investments in safer care or in requiring better training for clinicians or teams. Serious preventable adverse outcomes-while still surprisingly frequent-were uncommon for any single patient. Moreover, they persisted even in centres where simulation was used heavily, in part because of difficulties in reforming the systems of clinical work to match what was being taught. Embedded cultures and structures proved very resistant to change.

Studies showed that simulation training was in fact better for improving certain skills, but not all. Studies of simulation to address complex team behaviours were underpowered and targeted only one shot change. Long term multicentre studies and randomised controlled trials were never funded. No one was willing to pay the extra costs, even for few applications that had proven benefits, nor were they willing to invest in systematic and persistent applications of simulation to achieve enduring improvement. In truth, training continued to play second fiddle to the inexorable demands for clinical service. Because of this, the few centres that valiantly tried to fully integrate simulation training across the different dimensions went broke.

Professional societies, specialty boards, accrediting bodies, and regulators failed to act, even when there was modest evidence of benefit. For the professions, reforming training and practice took a back seat to demands regarding payment for care. Mandatory programmes of simulation training were strongly opposed by existing practitioners cautious of new requirements. They were also opposed by hospitals and training programmes unwilling to bear the costs and difficulties of completely reorganising their systems of training. Moreover, attempts to integrate simulation became embroiled in turf battles between different health care disciplines, between specialties, and between the different accrediting and regulatory bodies. 


\section{Text box 1: Key messages}

- Systematic training and assessment of health care personnel should become a major priority of the health care system.

- Simulation has a multitude of applications categorised by 11 dimensions.

- Simulation training will be applied not only to individuals, but more importantly also for crews, teams, work units, and organisations.

- Simulation will be an important "bottom up" tool for creating and maintaining a culture of safety and for fostering changes in work procedures and systems.

- Simulation will facilitate criterion based competency driven clinical training and practice.

- Costs of simulation based training will vary widely depending on the details of the application.

- Benefits of simulation applications may be hard to measure, especially those that will probably involve long term cumulative effects.

- Key drivers of the simulation vision include the public, liability insurers, professional societies, accrediting organisations, and governmental regulatory agencies.

There were some famous cases where poor training and skill led to clinical catastrophe, which made simulation training attractive to risk managers and insurers. But there was also a handful of malpractice cases that alleged negative or faulty simulation training as contributory to negligence. Most such allegations were rejected by the courts or successfully defended, but a few were sustained, with large monetary damages against simulation centres. Insurance for the simulation centres became expensive and hard to get, and malpractice insurers eliminated the discounts they had once offered for simulation training.

\section{THE VIEW AHEAD FROM 2004: WHICH HISTORY WILL COME TO PASS?}

One thing about guessing the history of the future is that the real history will almost certainly be different than either of these extremes, with an intermediate outcome being the most likely. In addition, different locales are likely to end up at different intermediate positions, depending on their unique local characteristics. Simulation for health care is nearly at the end of 20 years of steady progress, placing it perhaps at the "end of the beginning" stage (to quote Winston Churchill"). The fate of simulation as a means to a revolutionary change in health care is approaching a "tipping point ${ }^{\prime \prime 61}$ that will resolve itself strongly in the direction of one of these alternate histories over the next 10 years, although it will then take another decade to evolve fully.

The simulation community must educate the public and the implementing agencies on the vision of improved patient safety using the tool of simulation. The simulation community must also provide the core leadership in developing standards for simulators, curricula, and simulation centres. While we may never have unequivocal evidence of simulation's benefits equivalent to multiple randomised clinical trials, we should assemble the evidence where we can, and be

*In November, 1942, speaking after the second battle of El Alamein, Churchill said: "Now this is not the end. It is not even the beginning of the end. But it is, perhaps, the end of the beginning."
Text Box 2: Areas for further research and

\section{development}

- Integrating different types of simulation across different dimensions of applications, purposes, and target populations.

- Assessing the impact or benefit of simulation based training across the various dimensions.

- Developing applications for units of participation larger than clinical teams (complete work units, entire health care organisations).

- Establishing bench marks and criteria for competency based performance assessment using simulation.

- Investigating fundamental aspects of human performance in health care using simulation.

- Use of simulation for usability testing of medical devices and patient care processes, if possible at an early, prototype stage, and before deployment.

forthright in our drive to move forward where possible without ironclad proof. The current generation of leaders in health care and in medical simulation will be the key players in determining which direction the future history will actually take. As the Grateful Dead put it: "History's page will be neatly carved in stone. The future's here, we are it, we are on our own." (from "Throwing Stones," 1982; Lyrics, John P. Barlow; Music, Bob Weir; played by The Grateful Dead).

\section{REFERENCES}

1 Rochlin G, La Porte T, Roberts K. The self-designing high reliability organization: aircraft carrier flight operations at sea. Naval War College Rev 1987;42:76-90.

2 Roberts K. Some characteristics of high reliability organizations. Organizational Science 1990;1:160-77.

3 Gaba D. Structural and organizational issues in patient safety: a comparison of health care to other high-hazard industries. California Management Review 2001;43:83-102.

4 Kohn L, Corrigan J, Donaldson M. To err is human: building a safer health system. Washington, DC: National Academy Press, 1999.

5 Committee on quality of health care in America. Crossing the quality chasm: a new health system for the 21 st century. Washington, DC: National Academy Press, 2001.

6 Sutnick Al, Stillman PL, Norcini JJ, et al. ECFMG assessment of clinical competence of graduates of foreign medical schools. Educational Commission for Foreign Medical Graduates. JAMA 1993;270:1041-5.

7 Ziv A, Ben-David MF, Sutnick Al, et al. Lessons learned from six years of international administrations of the ECFMG's SP-based clinical skills assessment. Acad Med 1998;73:84-91.

8 Gaba DM, Howard SK, Flanagan B, et al. Assessment of clinical performance during simulated crises using both technical and behavioral ratings. Anesthesiology 1998;89:8-18.

9 Schwid HA, Rooke GA, Carline J, et al. Evaluation of anesthesia residents using mannequin-based simulation: a multi-institutional study. Anesthesiology 2002;97: 1434-44.

10 Fletcher G, Flin R, McGeorge $\mathrm{P}$, et al. Anaesthetists' non-technical skills (ANTS): evaluation of a behavioural marker system. $\mathrm{Br} J$ Anaesth 2003;90:580-8.

11 Boulet JR, Murray D, Kras J, et al. Reliability and validity of a simulationbased acute care skills assessment for medical students and residents. Anesthesiology 2003;99:1270-80.

12 Stefanich L, Cruz-Neira C. A virtual surgical simulator for the lower limbs. Biomed Sci Instrum 1999;35:141-5.

13 Krummel TM. Surgical simulation and virtual reality: the coming revolution. Ann Surg 1998;228:635-7.

14 Meier AH, Rawn CL, Krummel TM. Virtual reality: surgical application challenge for the new millennium. J Am Coll Surg 2001;192:372-84.

15 Howard S, Gaba D, Smith B, et al. Simulation study of rested versus sleep deprived anesthesiologists. Anesthesiology 2003;98:1345-1355.

16 Chopra V, Bovill J. Evaluation of a patient monitor using an anaesthesia simulator [abstract]. Anesthesiology 1995;83:A1064.

17 Agutter J, Drews F, Syroid N, et al. Evaluation of graphic cardiovascular display in a high-fidelity simulator. Anesth Analg 2003;97:1403-13.

18 Helmreich RL, Merritt AC, Wilhelm JA. The evolution of crew resource management training in commercial aviation. Int J Aviat Psychol 1999;9:19-32. 
19 Ruffell-Smith H. A simulator study of the interaction of pilot workload with errors, vigilance, and decisions. Washington, DC: National Aeronautics and Space Administration, 1979 January, 1979, Report No NASA TM-78482)

20 Billings CE, Reynard WD. Human factors in aircraft incidents: results of a 7 year study. Aviat Space Environ Med 1984;55:960-5

21 Gaba DM, Howard SK, Fish KJ, et al. Simulation-based training in anesthesia crisis resource management (ACRM): a decade of experience. Simulation and Gaming 2001;32:175-93.

22 Cooper J, Vogt J, Simon R, et al. Team training for healthcare administrators using full environment simulation [abstract]. Albuquerque, New Mexico: International Meeting on Medical Simulation, January, 2004.

23 Jensen R, Biegelski C. Cockpit resource management. In: Jensen R, ed. Aviation psychology. Aldershot: Gower Technical, 1989:176-209.

24 Weiner E, Kanki B, Helmreich R. Cockpit resource management. San Diego: Academic Press, 1993.

25 Satava RM. Emerging medical applications of virtual reality: a surgeon's perspective. Artif Intell Med 1994;6:281-8.

26 Satava RM. Surgical education and surgical simulation. World J Surg 2001;25:1484-9.

27 Bloom MB, Rawn CL, Salzberg AD, et al. Virtual reality applied to procedural testing: the next era. Ann Surg 2003;237:442-8.

28 Lefterie GS. How virtual reality may enhance training in obstetrics and gynecology. Am J Obstet Gynecol 2002; 187:S37-40.

29 Pittini R, Oepkes D, Macrury K, et al. Teaching invasive perinatal procedures: assessment of a high fidelity simulator-based curriculum. Ultrasound Obstet Gynecol 2002; 19:478-83.

30 Wang Y, Chui C, Lim H, et al. Real-time interactive simulator for percutaneous coronary revascularization procedures. Computer Aided Surgery 1998:3:211-27.

31 Cotin S, Dawson SL, Meglan D, et al. ICTS, an interventional cardiology training system. Stud Health Technol Inform 2000;70:59-65.

32 Gaba D, DeAnda A. A comprehensive anesthesia simulation environment: recreating the operating room for research and training. Anesthesiology 1988:69:387-94.

33 Rall M, Gaba D. Patient simulators. In: Miller R, ed. Anesthesia, 6th ed. New York: Elsevier, 2004; (in press).

34 Schwid H. A flight simulator for general anesthesia training. Comput Biomed Res 1987;20:64-75.

35 Good ML, Gravenstein JS. Anesthesia simulators and training devices. Int Anesthesiol Clin 1989;27:161-8.

36 Murray WB, Foster PA. Crisis resource management among strangers: principles of organizing a multidisciplinary group for crisis resource management. J Clin Anesth 2000;12:633-8.

37 Hegarty $M$, Bloch $M$. The use of simulators in intensive care training. Curr Anaesth Crit Care 2002;13:194-200.

38 Lighthall GK, Barr J, Howard SK, et al. Use of a fully simulated intensive care unit environment for critical event management training for internal medicine residents. Crit Care Med 2003:31:2437-43.

39 Small S, Wuertz R, Simon R, et al. Demonstration of high-fidelity simulation team training for emergency medicine. Acad Emerg Med 1999;6:312-23.

40 McLaughlin SA, Doezema D, Sklar DP. Human simulation in emergency medicine training: a model curriculum. Acad Emerg Med 2002;9:1310-18.
41 Reznek M, Harter P, Krummel T. Virtual reality and simulation: training the future emergency physician. Acad Emerg Med 2002;9:78-87.

42 Reznek M, Smith-Coggins R, Howard S, et al. Emergency medicine crisis resource management (EMCRM): pilot study of a simulation-based crisis management course for emergency medicine. Acad Emerg Med 2003:10:386-9.

43 Sica G, Barron D, Blum R, et al. Computerized realistic simulation: a teaching module for crisis management in radiology. Am J Roentgenol 1999; 172:301-4.

44 Miller G. The assessment of clinical skills/competence/performance. Acad Med 1990;65:S63-7

45 Helmreich $\mathbf{R}$, Schaefer $\mathrm{H}$. Team performance in the operating room. In Bogner $M$, ed. Human error in medicine. Hillsdale, NJ: Lawrence Erlbaum Associates, 1994:225-53.

46 Mohr J, Batalden PB. Improving safety on the front lines: the role of clinical microsystems. Qual Saf Health Care 2002; 11:45-50.

47 Nelson EC, Batalden PB, Huber TP, et al. Microsystems in health care. Part 1 Learning from high-performing front-line clinical units. Jt Comm J Qual Improv 2002;28:472-93.

48 Halamek L, Kaegi D, Gaba D, et al. Time for a new paradigm in pediatric medical education: teaching neonatal resuscitation in a simulated delivery room environment. Pediatrics. 2000;106: e45 (on-line))

49 Tsai TC, Harasym PH, Niissen-Jordan C, et al. The quality of a simulation examination using a high-fidelity child manikin. Med Educ 2003;37/suppl 1):72-8.

50 Stillman P, Swanson D. Ensuring the clinical competence of medical school graduates through standardized patients. Arch Intern Med 1987; 147:1049-52.

51 Barrows HS. An overview of the uses of standardized patients for teaching and evaluating clinical skills. Acad Med 1993;68:443-51.

52 Beullens J, Rethans J, Goedhuys J, et al. The use of standardized patients in research in general practice. Fam Pract 1997;14:58-62.

53 Thompson S, Neal S, Clark V. Clinical risk management in obstetrics: eclampsia drills. Qual Saf Health Care 2004;13:127-9.

54 Cooper J, Barron D, Blum R, et al. Video teleconferencing with realistic simulation for medical education. J Clin Anesth 2000;12:256-61.

55 Schwid H, O'Donnell D. The Anesthesia Simulator Consultant: simulation plus expert system. Anesthesiol Rev 1993;20:185-9.

56 Satava RM, Gallagher AG, Pellegrini CA. Surgical competence and surgical proficiency: definitions, taxonomy, and metrics. J Am Coll Surg 2003; 196:933-7

57 Satava RM, Cuschieri A, Hamdorf J. Metrics for objective assessment. Surg Endosc 2003;17:220-6.

58 Salas E, Fowlkes J, Stout R, et al. Does CRM training improve teamwork skills in the cockpit? Two evaluation studies. Human Factors 1999;41:326-43.

59 Salas E, Burke C, Bowers $C$, et al. Team training in the skies: does crew resource management (CRM) training work? Human Factors 2001:43:641-74

60 Wildavsky A. Doing better and feeling worse: the political pathology of health policy. Daedalus 1977:105-23.

61 Gladwell M. The tipping point: how little things can make a big difference. New York: Little Brown, 2002 